\title{
Gait Programming for Multi-Legged Robot Climbing on Walls and Ceilings
}

\author{
Jinwu Qian, Zhen Zhang and Li Ma \\ Dept. of Precision Mechanical Engineering, Shanghai University, Shanghai \\ China
}

\section{Introduction}

At present, the first-step surface adhesion of most wall-climbing robots depends on some outside force, which is provided either by human operators or by such a special-purpose mechanical device as a manipulator. This not only restricts many special applications of wall-climbing robots. But also makes it impossible for the robot to fulfil automatic operations continually on walls with surface intersections.

With the steady expansion of possible application areas of wall-climbing robots, it is natural to make higher expectations on the robots' performance, i.e., automated transit walking ability from ground to wall, from one wall to another, or from wall to ceiling.

Japanese researchers take the lead to explore that kind of wall-climbing robot in recent years. The mechanism configurations of the two prototypes developed fall into the deformable framework category [Ikeda, 1991] and the multi-bodied wheel type [Sato, 1992]. However, the above two robot mechanisms have some major disadvantages on mobility, irregular obstacle negotiation and on wall shape adaptability, because they do not possess

$\varepsilon$ independently actuated leg mechanism. Therefore, it is reasonable to think that multi-legged ơ wall-climbing mechanism is a better alternative [Qian,1993].

C Compared with other forms of mobile robot, multi-legged robot is a suitable option to realize transition motions. Each independently-driven leg's foothold can be selected in order ธे to adapt complicated environment.
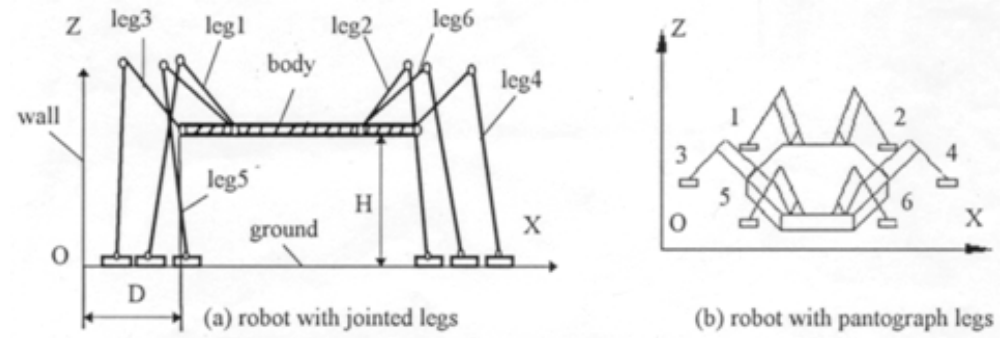

(b) robot with pantograph legs

Figure 1 Multi-legged robot

Many theoretical and practical problems are to be solved before the actual implementation of a multi-legged wall-climbing robot. Among them is the ground-to-wall gait programming that is investigated in this paper, and a six-legged wall-climbing robot is discussed here as an example. 
Wall climbing robots have extensive potential applications on tall buildings, large storage tanks and vessel bodies. Typical automatic operations are cleaning, painting and detecting. After years of research, various types of vacuum-adhering wall-climbing robots have been developed and reported, which can be characterized primarily as the sliding-sucker type (Nagastuka, 1996; Men, 1994), the fixed-sucker tracked type(Nagastuka, 1996) and the fixedsucker walking beam type (Kroczynski, 1987; Ikeda, 1989; Chen, 1997).

Each type of wall-climbing robot mentioned above has its own distinct features. The slidingsucker wheeled type and the fixed-sucker tracked type, with a comparatively high velocity, lack the ability to overcome obstacles. The fixed-sucker walking beam type can carry a relatively heavy payload, although it climbs at a rather slow speed. As none of them can adapt to walls with irregular obstacles, still another type, multi legged robots, have come into the sight of robotic researchers (Hirose, 1992; Luk, 1991).

Along with the ability to negotiate obstacles and adapt to different wall shapes, the multilegged robot is also capable of climbing from the ground to the wall.

In the configuration design for a six-legged wall-climbing robot, the following questions must be answered first:

Is it possible to find a simple measurement to evaluate legged wall gaits, so that the traditional approaches for the ground gait analysis can be referred to?

As the number of possible six-legged gaits is large, which gait should be applied to the robot climbing on vertical walls or on ceilings?

Between insect-type and crab-type configurations of leg stroke layouts, which is better suited for a wall-climbing robot?

Among the possible duty factors of $1 / 2 \leq \beta<1$ for the static locomotion, which is more suited from the viewpoint of speed and safety?

To answer these questions, the authors propose geometric measurements related to support patterns to describe the overturn resistance capability of multi-legged robots climbing on both vertical wall and ceilings. Optimal regular periodic wall gaits and ceiling gaits are calculated and selected. Comparisons on overturn resistance capability are made for crabtype and insect-type of leg-stroke layouts. A reasonable duty factor for both speed and safety is recommended.

\section{Simplification of Robot-environment Modelling}

As is known to all, wall-climbing robot needs suction caps to adhere on the wall, which could be either vacuum-activated or magnet-activated, and which are pivoted in the foot ends. Suction cups are referred to as pads which bring complications in the gait programming process of legged robots.

In order to simplify the robot-environment modelling, we imagine taking off pads from robot legs virtually, at the same time put some constraint conditions which keep the legs' motion realistic as if pads are still on. We call this method virtual pad removal.

When pads were taken off from legs, we must introduce other new ideas: virtual ground and virtual wall, which can be understand clearly when considering the robot walking on the level ground of the vertical wall surface.

As the actual robot is moving on the level ground with suction cups, the ankle joints are somewhat like walking on another surface parallel to the level ground. That surface is referred to as virtual ground. Virtual wall can be defined in the same way. 
After virtual pad removal and virtual surface concept are introduced, we can investigate the multi-legged robot with no suction cups moving on virtual surfaces. While the effect is all the same as we investigate robot with suction cups which moves from real ground to real wall, we make the robot-environment modelling much simpler.

\section{Implementation of Transition Gait from Ground to Wall}

Compared with both the ground-walking gait [Qian, 1998] and the wall-climbing gait programming [Qian, 1997], the ground-to-wall transition walking has its unique features. From the starting and the ending status (Fig.2) of the transition gait, it can be seen that the transition movement changes not only the foothold support plane of the robot, but the body frame orientation in the world frame as well.

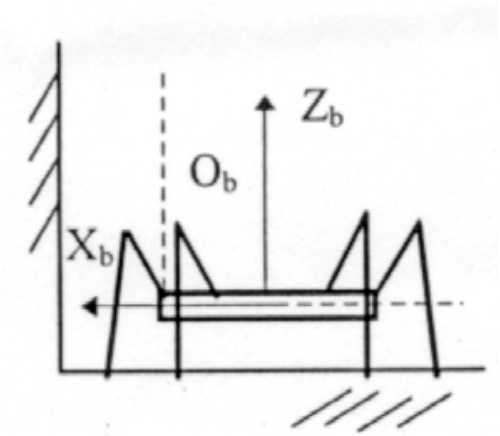

(a) Starting moment of transit gait

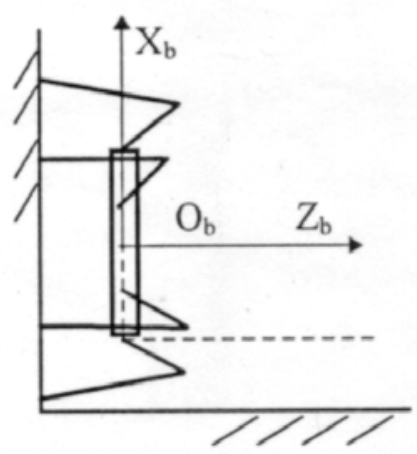

(b) Ending moment of transit gait

Figure 2. Transit gait of robot from ground to wall

The problem that has to be solved first is to decompose the whole seemingly complicated transition walking process into combinations of simpler motions that are easy to describe and realize.

In order to free ourselves from seeking the inherent law which governs the transition gait, the authors just decompose it, from the engineering viewpoint, into some combinations of two basic motions which are defined as leg transferring and body pitching.

In leg transferring, the robot's body orientation keeps unchanged, only one or more leg transfers from one foothold to another. While in body pitching, all footholds of supporting legs remain the same, and the body fulfils incremental planar movement along some definite trajectory because of leg joints' motions. The two basic motions are described below in details.

\subsection{Leg transferring}

When the robot is near the corner of ground-wall-intersection or in the course of transition movement, the leg transferring has three different situations according to the features of the starting and the ending foothold positions:

(1) ground-to-ground transfer

(2) ground-to-wall,

(3) wall-to-wall transfer. 
Each of them can be further divided into three phases---leg lifting, leg transfer and leg lowering. The projections of foot trajectories in YOZ plane of the world frame (Fig.3) are shown in Fig. 4. It should be noted that the foot trajectories here are only for conceptual understanding and may be modified for better dynamic behavior.

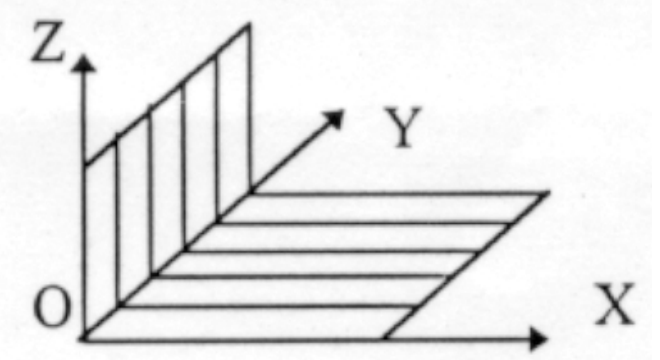

Figure 3. World coordinate system

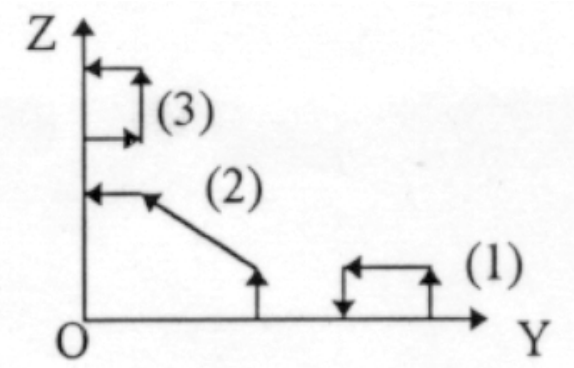

Figure 4. Foot tip trajectories

\subsection{Body pitching}

Several legs are used in body pitching motion according to gait features. Considering the starting and the ending body orientations in the transit gait which are shown in Fig.2(a) and Fig.2(b), it can be deduced that the most efficient pitching method for the robot to adopt is rotating with two virtual end limitations. It means that in the course of body pitching, the trajectory of one specific point on the front part of the body is parallel to the wall surface, while the trajectory of another specific point in the rear part of the body is parallel to the ground, as if the front and the rear end of the robot body are always moving in a virtual vertical guideway and in a virtual horizontal guideway respectively, as shown in Fig.5.

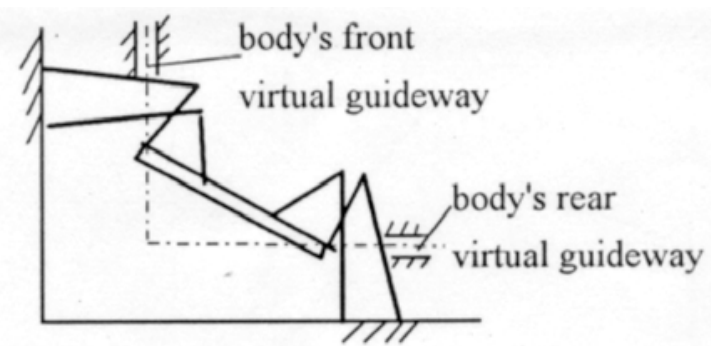

Figure 5. Rotation method of two virtual and limitation 


\subsection{Strategy of transit gait}

After single-leg transferring and body pitching are defined, the transition gait algorithm can be programmed by combining them in a reasonable manner. Here we consider a six-legged robot model with jointed legs.

Step1. Starting phase, at this moment the robot is standing on the ground with six legs supporting. Body height from the ground and the distance from the body's front end to the wall are $\mathrm{H}$ and $\mathrm{D}$ respectively, referring to Fig.6(a);

Step2. Calculate highest front-leg's foothold on the wall;

Step3. Transfer front leg from the ground to the wall, referring to Fig. 6(b);

Step4. Make body pitching motion, until one or more legs are near the rear boundary of their working envelops. Note down their sequential number, referring to Fig. 6(c);

Step5. Calculate and seek new footholds of legs that are in the boundary of working envelops. Then do leg transferring one by one, referring to Fig. 6(d);

Step6. If accumulating pitching angle is smaller than 90, go to step 4, otherwise go to step 7 .

Step7. Seek legs that are still on the ground, and transfer them to the wall surface, referring to Fig.6(e).

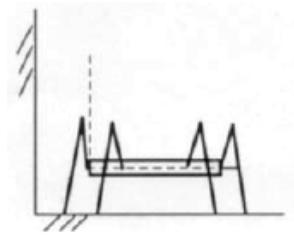

a)

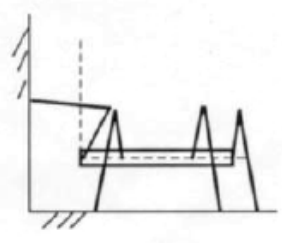

b)

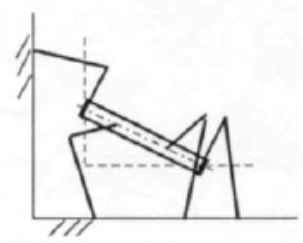

c)

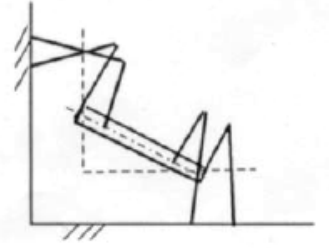

d)

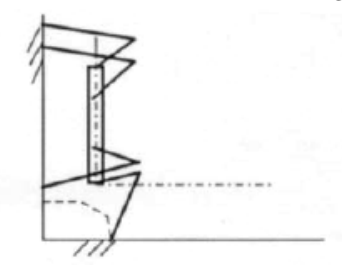

e)

Figure 6. Graphic expression of transit gait

\section{Modules and Flowchart of Computer Simulation Program}

To verify the feasibility and effectiveness of transit gait strategy and algorithm, we made a kinematics simulation on a computer. Turbo $C$ language is used to write software whose structural modules are shown in Figure 7. The design considerations and main flowcharts of the simulation software are discussed briefly as follows.

\subsection{Module for coordinate transformation}

The leg-tip positions and the body pose are given in world coordinate system OXYZ, which coincides with the CRT coordinate system $\mathrm{O}, \mathrm{X}, \mathrm{Y}, \mathrm{Z}$, for convenience, while the working envelopes of legs are given in body coordinate system. Sometimes the solving of a part of the kinematics problems of transit gait is more convenient in body coordinate system. For coordinate transformation of robot position and orientation between different frames, coordinate transformation module is designed. 


\subsection{Geometrical graphics module}

This module includes a robot modeling part and an environment modeling part. The environment structure adopts rectangular box-like geometry to simulate ground, wall and ceiling. The shape of the robot is simplified as line geometry combination.

\subsection{Robot animation module}

The effect of animation utilizes eye image detainment phenomenon in visual neural network. For obtaining better animation effect, two graphics buffers are used in this module. The alternating display and refreshment of the two graphics buffers can be done by such internal functions as "setactivepage" and "setvisualpage". Before that one should set VGA graphics to medium resolution mode in graphics initialization block of the software.

In addition, if the exchange frequency of display page is too high, the CRT screen will flicker. Delay function can be used to fix this problem.

\subsection{Single-leg transfer module}

This module is designed for the leg tip to transfer from an old foothold to a new one along a given trajectory. Before every incremental movement of a leg-tip an inverse kinematics subroutine is run once and the whole robot is redrawn.

\subsection{Body pitching module}

As stated earlier, six legs are in support phase when the robot body rotates. In the body pitching process, the screen coordinates of legs do not change. On the other hand, when the leg joints are activated, the robot body pose will change in a predetermined way. In the program, inverse kinematics subroutine is run again and again according to body pose modification. In every incremental rotation the whole robot is redrawn.

\subsection{Kinematics module}

This module undertakes the task of acquiring of new footholds, checking-up of foot tips that are near their working envelopes and solving inverse kinematics. It should be noted that when multi-solution problem of inverse kinematics emerges the program will make the right choice by checking them with the parameters of the previous.

The main flowchart of the simulation software is shown in Fig.7 through Fig.10.

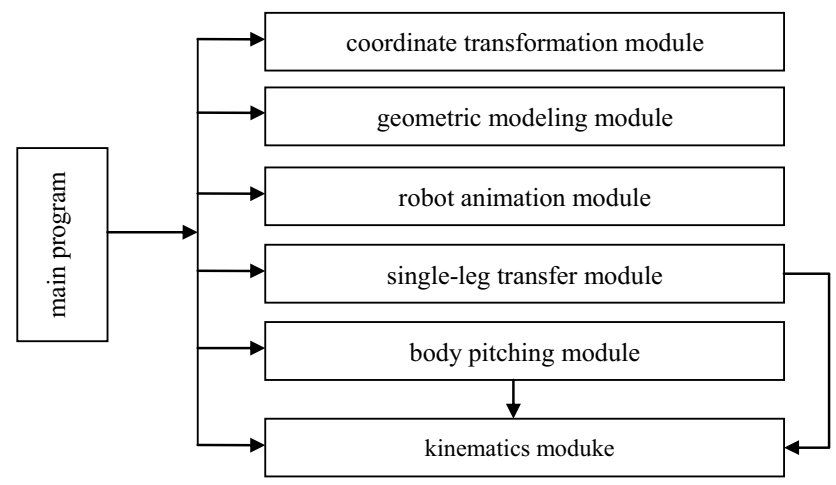

Figure 7. Modular structure of kinematics simulation software 


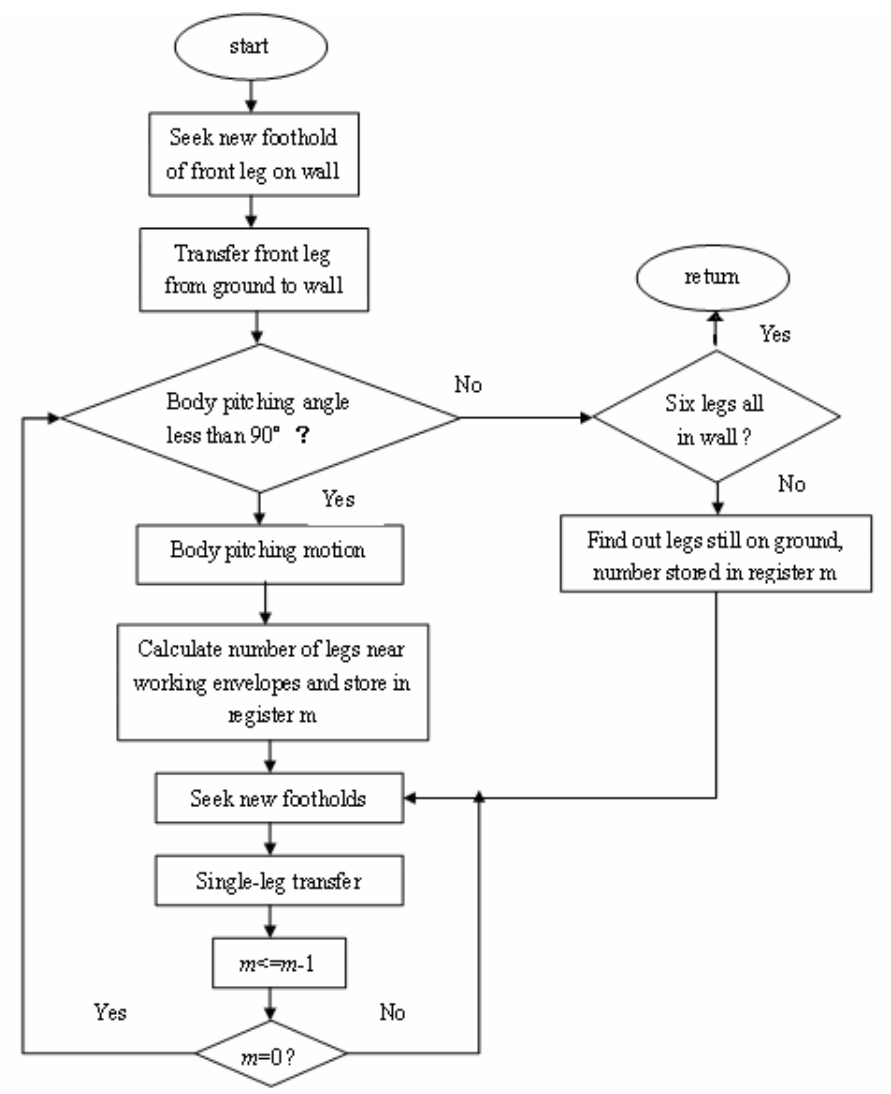

Figure 8. Flowchart of main program for transit gait

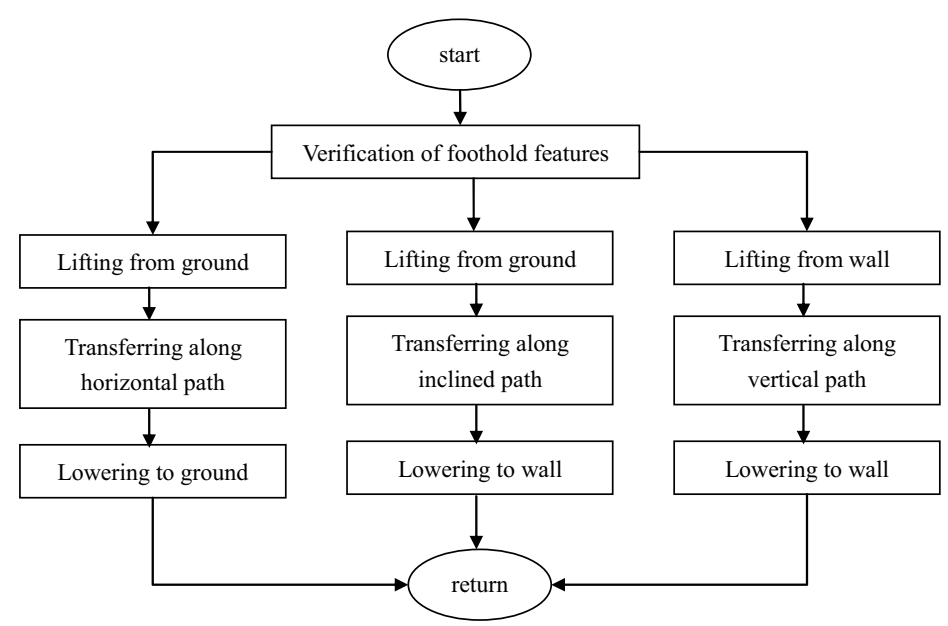

Figure 9. Flowchart of single-leg transfer 


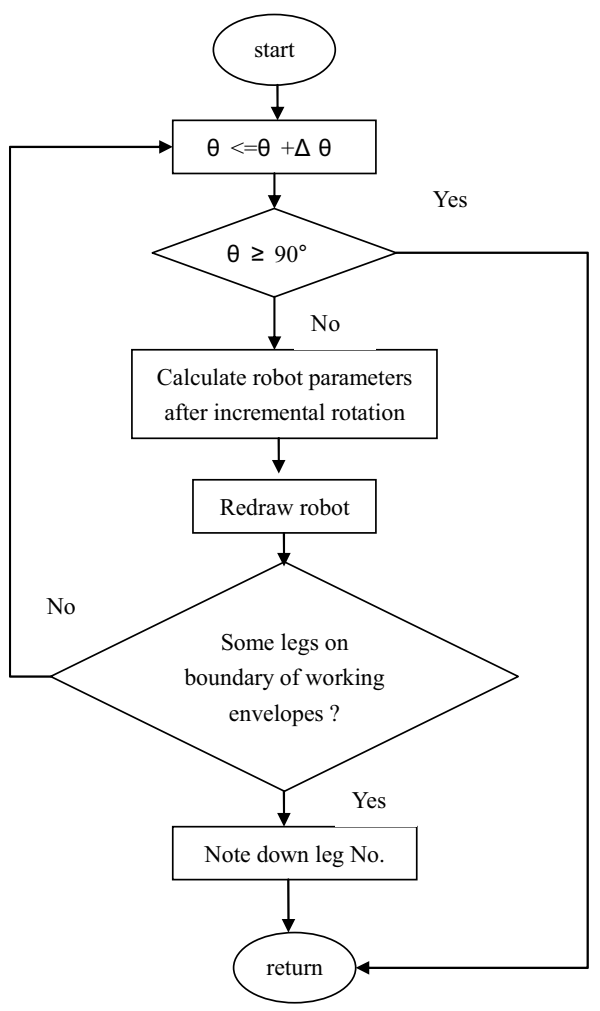

Figure 10. Flowchart of body pitching motion subroutine

\section{Feasibility and Rapidity of Transition Gait}

Whether the ground-to-wall transition gait can be realized or not when the dimensions of the robot body and the position between the robot and the environment (ground and wall) have changed, and how to select these parameters reasonably to perform transition motion rapidly are important issues, which have to be investigated in detail, here we only give the results [Gu,1997],referring to Fig.11.

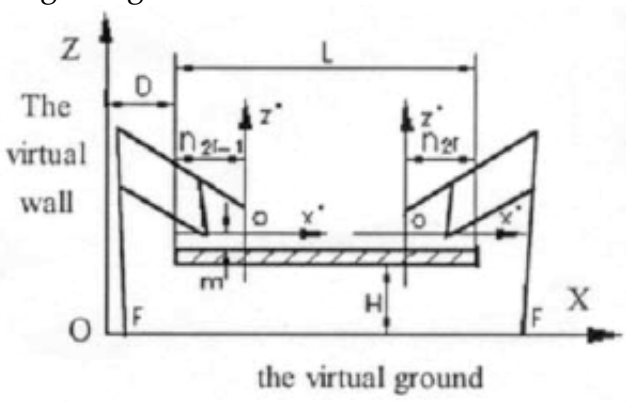

Figure 11. Simplified model of the actual mechanism 


\subsection{Effect of robot body length on transition gait}

When assigning $\mathrm{D}$ and $\mathrm{H}$ to specific values, the times of adjusting the robot body increase little by little with the increase of the robot body's length $\mathrm{L}$. If the length $\mathrm{L}$ goes beyond a certain value, the adjusting times will become very large. If the length is too large, the robot can not pitch either in the beginning or after pitching the robot body a certain angle because of the pad constrained conditions of the even-numbered legs, it will fail to fulfil ground-wall transitional motion. So after the dimensional requirements of assembling other components are assured, the shorter the length of the robot body, the more rapidly the robot can move from the ground to the wall.

\subsection{Effect of distance $D$ on transition gait}

When distance $\mathrm{D}$ increases, the feasibility of the ground-wall transitional motion also alters. If $\mathrm{D}$ is too large, some legs find difficulties in climbing the wall in the beginning or when the accumulative pitching angle is large enough, the even-numbered legs will be suspended (the leg can't reach both the ground and the wall) or will be refrained by their pads, and leads to the failure of the transition motion. On the other hand, if the distance D is too small, the robot body's pitching motion is difficult at the start due to the pad constrained conditions of the odd-numbered legs. So the distance D should be assigned a middle value in its variable limitation determined by the leg structure.

\subsection{Effect of distance $\mathrm{H}$ on transition gait}

The effect of distance $\mathrm{H}$ on feasibility of transition gait is similar to that of distance D. If the distance $\mathrm{H}$ is too large, it is difficult for the robot to pitch its body in the beginning because the sharp angles of the even-numbered legs act on the leg mechanism seriously. If the distance $\mathrm{H}$ is too small, the leg will interfere with the ground easily after pitching the robot body to a certain angle so that results in the failure of the ground-wall motion transitional motion.

\section{Gait Programming for Climbing on Wall}

Gait programming in climbing process is one of basic issues for the multi-legged robot working on vertical walls and ceilings.

\subsection{Nomenclature}

$\mathrm{C}_{s p}$-Anti-Overturn Coefficient of a given support pattern, while the robot is adhering in an inclined wall surface

$\mathrm{C}_{G T}-$ Anti-Overturn Coefficient of a regular periodic gait

$\mathrm{D}_{s p}$-Anti-Overturn Distance of a given support pattern

$\mathrm{D}_{\mathrm{GT}}-$ Anti-Overturn Distance of a regular periodic gait

$\mathrm{F}_{s}$ - suction force of a single leg (or suction cup)

$H$-perpendicular distance from the robot's center of gravity to the wall surface

$M$ - weight moment, $M=W \cdot H$

$M_{\max }$ - maximal weight moment, that is $M_{\max }=\left\{M \mid \mathrm{N}_{j \min }=0\right\}$

$\mathrm{N}_{i}$ - normal reaction force acted on sucker $\mathrm{i}$ by the wall surface

oxyz-robot body coordinate system with its origin in the robot's center fo gravity, $x$ axis directs to the locomotion direction, $\mathrm{z}$ axis directs perpendicularly away from the wall surface 
$x_{i,} y_{i}-$ coordinates of sucker I in body frame oxyz

$a-$ angle between the locomotion direction and the horizontal base line

$\beta$-duty factor of a regular periodic gait for a legged robot

$\theta$-angle of the inclined wall surface with respect to the horizontal plane, $\theta=0$ for ceilings, $\theta=90^{\circ}$ for vertical walls

$\varphi_{i}, \varphi_{i}{ }^{*}$ - relative phase of leg $i$ and its optimal value

\subsection{Nomenclature Assumptions on robot model}

In order to simplify the gait programming task of multi-legged robots climbing on walls and ceilings, following assumptions are proposed,

1) C.G. consistency assumption: in the robot motion cycle, the position of the robot's center of gravity remains the same with respect to the body coordinate system.

2) Suction force consistency assumption: the suction force of each sucker is the same.

3) Height consistency assumption: in the locomotion cycle, the distance of the robot's center of gravity away from the wall surface remains the same.

4) Small support area assumption: the foothold of the robot or the size of the sucker is relatively small compared with the whole robot.

5) Rigidity assumption: the robot as a whole is much more rigid than the sucker's sealing ring that is made of rubber.

\subsection{Geometric measurement for overturn resistance capability}

As we know, the criterion to evaluate ground-walking gait is the stability margin, which was used in ground gait analysis (McGhee, 1968; Bessonov, 1973; Hirose, 1984; Qian, 1988). While in the scenario of a robot climbing on the wall, the direction of the gravitational force acted on the robot is quite different from that on the ground robot. But we would like to establish a new criterion to describe the safety margin of the wall-climbing robot, which is still based on the support pattern, as is the case of the stability margin for the groundwalking robot.

The safety of a wall-climbing robot is actually the payload reserve that the robot can carry, which can be further divided into Anti-slippage Capability Reserve and Anti-overturn. The Anti-slippage Capability Reserve is assured by properly choosing the suction cup material, the vacuity inside the suckers and the minimal number of adhering suckers. While the Antioverturn Capability Reserve is determined not only by the suction forces and the number of functioning suckers, but by their relative positions, i.e., the support patterns as well. So in gait analysis and optimal gait selection, it is reasonable to put more emphasis on Antioverturn Capability Reserve.

Following definition describes a geometric measurement for Anti-overturn Capability Reserve.

Definition 1: Anti-Overturn Distance (abbreviated as AOD) of a given support pattern, $D_{s p}$, is the maximal weight moment Mmax, divided by the suction force of a single sucker.

$$
\mathrm{D}_{\mathrm{sp}}=\frac{\mathrm{M}_{\max }}{\mathrm{F}_{\mathrm{s}}}
$$

From this definition, the Anti-overturn Capability Reserve can be calculated as

$$
n=\frac{D_{s p} F_{s}}{M}
$$


where $\mathrm{M}$ denotes the actual weight moment.

The following theorem deals with the calculation of AOD.

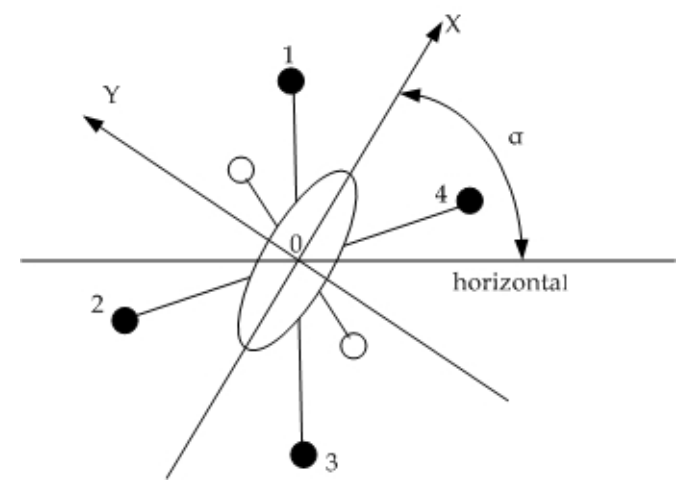

Figure 12. Legged wall robot side view with body frame attached (* Black circle denoted the suckers in support status)

Theorem 1: Given the foothold coordinates $\left(x_{i}, y_{i}\right)$ of adhering suckers in body frame oxyz and the climbing direction angle a as specified in Fig.12, the Anti-overturn Distance is calculated as

$$
D_{s p}=-1 / \min _{i=1-n}\left(r_{o i}\right)
$$

Where $n$ is the number of suckers in support status. $r_{o i}$ is the $i$ th element of vector $\mathbf{R}_{\mathbf{0}}$ :

$$
\mathbf{R}_{0}=\mathbf{A}^{-1} \mathbf{Q}_{0}
$$

Where $\mathrm{A}$ is a matrix related with the adhering suckers' positions, $\left(x_{i}, y_{i}\right)$, in body's coordinate system:

$$
\begin{gathered}
\mathbf{A}=\left[\begin{array}{ccccccc}
1 & 1 & 1 & \ldots & \ldots & \cdots & 1 \\
x_{1} & x_{2} & x_{3} & \ldots & \ldots & \ldots & x_{n} \\
y_{1} & y_{2} & y_{3} & \ldots & \ldots & \ldots & y_{n} \\
f_{41} & f_{42} & f_{43} & f_{44} & & & \mathbf{0} \\
f_{51} & f_{52} & f_{53} & & f_{55} & & \\
\vdots & \vdots & \vdots & & & \ddots & \\
f_{n 1} & f_{n 2} & f_{n 3} & \mathbf{0} & & & f_{n n}
\end{array}\right]_{n \times n} \\
f_{j 1}=\left(x_{2}-x_{j}\right)\left(y_{3}-y_{j}\right)-\left(x_{3}-x_{j}\right)\left(y_{2}-y_{j}\right) \\
f_{j 2}=\left(x_{3}-x_{j}\right)\left(y_{1}-y_{j}\right)-\left(x_{1}-x_{j}\right)\left(y_{3}-y_{j}\right) \\
f_{j 3}=\left(x_{1}-x_{j}\right)\left(y_{2}-y_{j}\right)-\left(x_{2}-x_{j}\right)\left(y_{1}-y_{j}\right) \\
f_{j j}=-\left(f_{j 1}+f_{j 2}+f_{j 3}\right) \\
j=4,5, \ldots, n
\end{gathered}
$$


And $\mathbf{Q}_{\mathbf{0}}$ is the unit load vector normalized to $\mathbf{M}$,

$$
\mathbf{Q}_{0}=(0,-\sin \alpha,-\cos \alpha, 0, \cdots, 0)_{n \times 1}^{T}
$$

Proof:

i)Three-sucker support pattern

Let's first consider the situation that three of the robot's suckers are in contact with the wall surface. In this support pattern, three equilibrium equations can be established for the static force system, Expressed in a compact matrix form, we have

$$
\left[\begin{array}{ccc}
1 & 1 & 1 \\
x_{1} & x_{2} & x_{3} \\
y_{1} & y_{2} & y_{3}
\end{array}\right]\left(\left\{\begin{array}{l}
N_{1} \\
N_{2} \\
N_{3}
\end{array}\right\}-\left\{\begin{array}{l}
F_{s} \\
F_{s} \\
F_{s}
\end{array}\right\}\right)=\left\{\begin{array}{c}
0 \\
-M \sin \alpha \\
-M \cos \alpha
\end{array}\right\}
$$

For the convenience of deduction, abbreviate Eq. (7) as

$$
\mathbf{A}(\mathbf{N}-\mathbf{F})=\mathbf{Q}
$$

Make the following substitution with $\mathbf{N}_{0}$ and $\mathbf{Q}_{0}$

$$
\mathbf{N}=M \mathbf{N}_{\mathbf{0}}, \mathbf{Q}=M \mathbf{Q}_{\mathbf{0}}
$$

Substitute into Eq (8)

$$
\mathbf{A}\left(M \mathbf{N}_{0}-F_{s} \mathbf{I}\right)=M \mathbf{Q}_{0}
$$

where I is an identity vector, From $\mathrm{Eq}(9)$, we can get

$$
\mathbf{N}_{0}=\frac{F_{S}}{M} \mathbf{I}+\mathbf{A}^{-1} \mathbf{Q}_{0}
$$

Denote

$$
\mathbf{R}_{0}=\mathbf{A}^{-1} \mathbf{Q}_{0}
$$

Substitute it into $\mathrm{Eq}(10)$

$$
\mathbf{N}_{\mathbf{0}}=\frac{F_{S}}{M} \mathbf{I}+\mathbf{R}_{\mathbf{0}}
$$

When $M$ increase and approaches $M_{\max }$, one of the normal reaction forces acting on suckers becomes zero,

$$
\min _{i=1-3}\left(N_{0 i}\right)=0
$$

Then, we can extract an algebra equation from $\mathrm{Eq}(12)$

$$
0=\frac{F_{S}}{M_{\max }}+\min _{i=1-3}\left(r_{0 i}\right)
$$


So the AOD of three-sucker support pattern is

$$
D_{s p}=\frac{M_{\max }}{F_{s}}=-\frac{1}{\min _{i=1-3}\left(r_{0 i}\right)}
$$

ii)Four-sucker support pattern

In four-sucker support pattern, three force equilibrium equations can also be combined into one matrix equation,

$$
\left.\left[\begin{array}{cccc}
1 & 1 & 1 & 1 \\
x_{1} & x_{2} & x_{3} & x_{4} \\
y_{1} & y_{2} & y_{3} & y_{4}
\end{array}\right] \cdot\left\{\begin{array}{l}
N_{1} \\
N_{2} \\
N_{3} \\
N_{4}
\end{array}\right\}-\left\{\begin{array}{l}
F_{S} \\
F_{S} \\
F_{S} \\
F_{S}
\end{array}\right\}\right)=\left\{\begin{array}{c}
0 \\
-M \sin \alpha \\
-M \cos \alpha
\end{array}\right\}
$$

$\mathrm{Eq}(14)$ is statically-indeterminate, that is, there are four unknowns in only three equations. But it could be solved after adding one deformation coordination constraint. From the rigidity assumption proposed earlier, under gravitational forces, the displacements of the robot's foot tips away from the wall can be considered to be distributed so that they are still in one plane.With the four-point co-plane standard equation and Hooke's Law considered, we get

$$
\left|\begin{array}{lll}
x_{1}-x_{4} & y_{1}-y_{4} & N_{1}-N_{4} \\
x_{2}-x_{4} & y_{2}-y_{4} & N_{2}-N_{4} \\
x_{3}-x_{4} & y_{3}-y_{4} & N_{3}-N_{4}
\end{array}\right|=0
$$

which can also be express as

$$
f_{41} N_{1}+f_{42} N_{2}+f_{43} N_{3}+f_{44} N_{4}=0
$$

Where

$$
\begin{gathered}
f_{41}=\left(x_{2}-x_{4}\right)\left(y_{3}-y_{4}\right)-\left(x_{3}-x_{4}\right)\left(y_{2}-y_{4}\right) \\
f_{42}=\left(x_{3}-x_{4}\right)\left(y_{1}-y_{4}\right)-\left(x_{1}-x_{4}\right)\left(y_{3}-y_{4}\right) \\
f_{43}=\left(x_{1}-x_{4}\right)\left(y_{2}-y_{4}\right)-\left(x_{2}-x_{4}\right)\left(y_{1}-y_{4}\right) \\
f_{44}=-\left(f_{41}+f_{42}+f_{43}\right)
\end{gathered}
$$

Combine $\mathrm{Eq}(16)$ with $\mathrm{Eq}(14)$ into one matrix equation

$$
\left.\left[\begin{array}{cccc}
1 & 1 & 1 & 1 \\
x_{1} & x_{2} & x_{3} & x_{4} \\
y_{1} & y_{2} & y_{3} & y_{4} \\
f_{41} & f_{42} & f_{43} & f_{44}
\end{array}\right] \cdot\left\{\begin{array}{l}
N_{1} \\
N_{2} \\
N_{3} \\
N_{4}
\end{array}\right\}-\left\{\begin{array}{l}
F_{S} \\
F_{S} \\
F_{S} \\
F_{S}
\end{array}\right\}\right)=\left\{\begin{array}{c}
0 \\
-M \sin \alpha \\
-M \cos \alpha \\
0
\end{array}\right\}
$$


Through substitutions and similar approaches used for the three-sucker support pattern, we obtain the AOD for four-sucker support pattern

$$
D_{s p}=\frac{M_{\max }}{F_{s}}=\frac{-1}{\min _{i=1-4}\left(r_{0 i}\right)}
$$

where $r_{0 i}$ is the $i$ th element or vector $\mathbf{R}_{0}$

$$
\begin{gathered}
\mathbf{R}_{\mathbf{0}}=\mathbf{A}^{-1} \mathbf{Q}_{\mathbf{0}} \\
\mathbf{A}=\left[\begin{array}{cccc}
1 & 1 & 1 & 1 \\
x_{1} & x_{2} & x_{3} & x_{4} \\
y_{1} & y_{2} & y_{3} & y_{4} \\
f_{41} & f_{42} & f_{43} & f_{44}
\end{array}\right]_{4 \times 4} \\
\mathbf{Q}_{\mathbf{0}}=\left\{\begin{array}{c}
0 \\
-\sin \alpha \\
-\cos \alpha \\
0
\end{array}\right\}
\end{gathered}
$$

iii) n-sucker support pattern

For an n-sucker support pattern, force equilibrium equations in matrix form can be written as

$$
\left[\begin{array}{cccc}
1 & 1 & \cdots & 1 \\
x_{1} & x_{2} & \cdots & x_{n} \\
y_{1} & y_{2} & \cdots & y_{n}
\end{array}\right]_{3 \times n}\left(\left\{\begin{array}{c}
N_{1} \\
N_{2} \\
. . \\
. . \\
. . \\
N_{n}
\end{array}\right\}_{n \times 1}-\left\{\begin{array}{c}
F_{S} \\
F_{S} \\
. \cdot \\
. . \\
. . \\
F_{S}
\end{array}\right\}_{n \times 1}\right\}_{n \times 1}
$$

Altogether (n-3) deformation coordination equations can be written as

$$
\begin{gathered}
\left|\begin{array}{ccc}
x_{1}-x_{j} & y_{1}-y_{j} & N_{1}-N_{j} \\
x_{2}-x_{j} & y_{2}-y_{j} & N_{2}-N_{j} \\
x_{3}-x_{j} & y_{3}-y_{j} & N_{3}-N_{j}
\end{array}\right|=0 \\
j=4, \cdots, n
\end{gathered}
$$

When similar approaches are applied to this scenario, Eq.(2) through Eq.(6) are obtained.

\section{End of Proof}

From Eq.(2)through Eq.(6), it is obvious that AOD only depends on the relative positions of adhering suckers when the robot is climbing in the direction angle of $\alpha$ during a gait cycle, In other words, AOD is a geometric measurement for anti-overturn capability reserve. 


\section{Analytical Results of AOD}

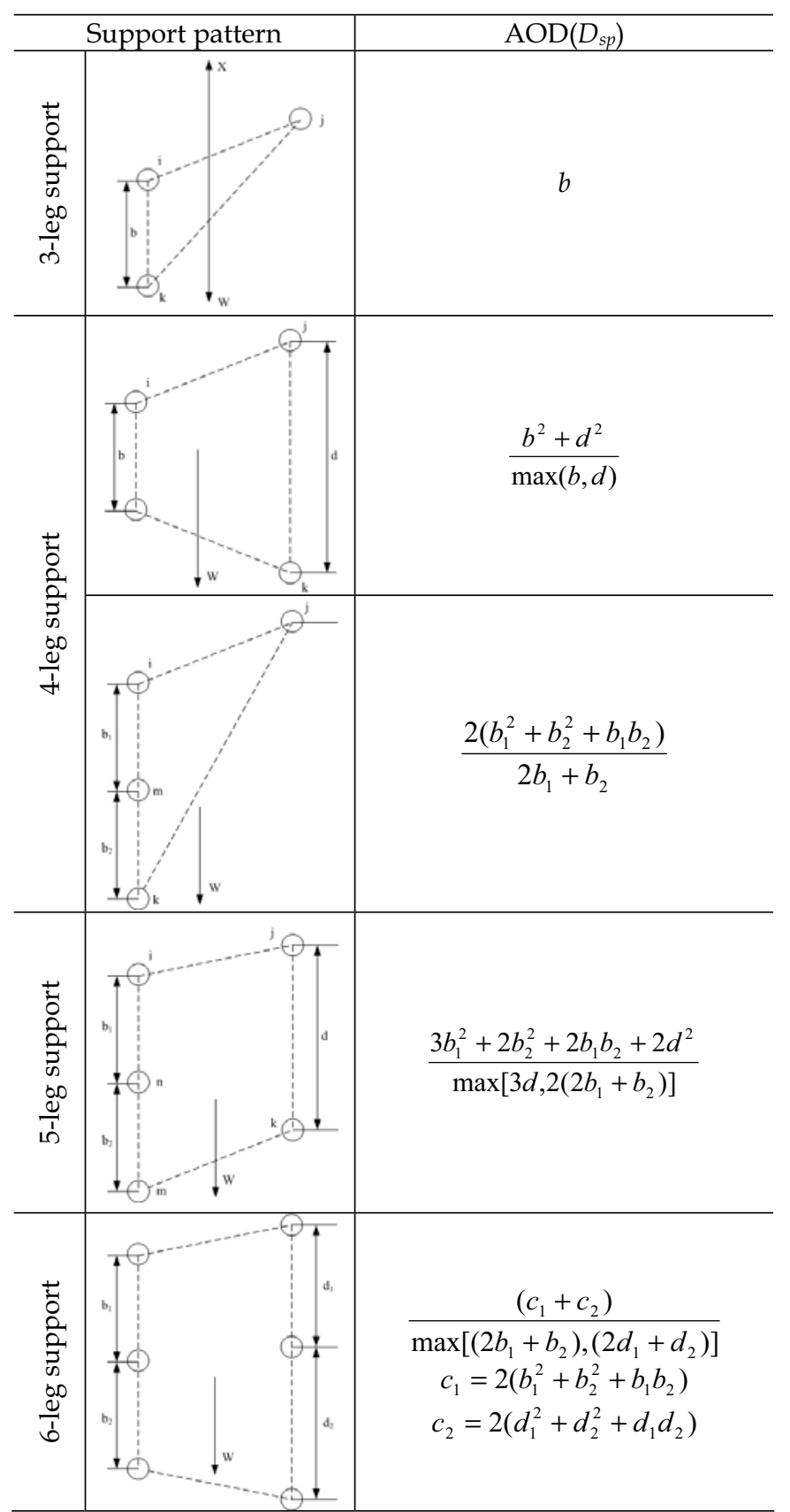

Table 1. Analytical solutions of AOD 
In the process of optimal gait programming using $\mathrm{AOD}$ as a goal function, repeated calculations of $D_{s p}$ are inevitable and time consuming since Eq.(3) calculates matrix inversion, which leads to difficulty for real-time gait programming or real-time safety monitoring of a legged robot climbing on wall surfaces with obstacles.

To overcome this problem, it is necessary to seek the analytical solutions for different support patterns in advance. When the analytical expressions of AOD are obtained, the great enhancement of gait programming efficiency becomes possible.

The procedures of calculating AOD are summarized as,

i) Establish three static force balance equations.

ii) Examine the number of suckers in support status. If functioning sucker number $n$ is greater than three, $(\mathrm{n}-3)$ deformation constraint requirements are established.

iii) Calculate the normal reaction force on the suckers by the wall, $N_{i}$, and determine the minimal of them, $N_{\min }$.

iv) Let $N_{\min }=0$, and get the expression of $D_{s p}$.

Using the robot model as shown in Fig.13, the analytical solutions of AOD for three-through six-leg support patterns are deduced following the above procedures. The results are listed in Table 1.

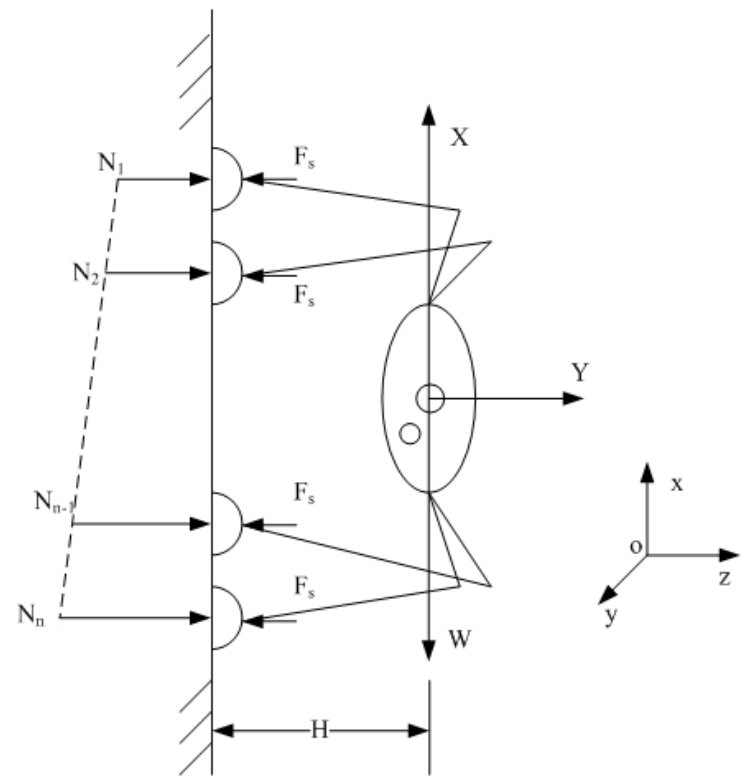

Figure 13. Legged wall robot side view with acting forces

\subsection{Optimal gaits on vertical wall}

Mathematical model

The geometric models of the robot's leg stroke layouts are depicted in Fig.14. The coordinate system in Fig.14 has been normalized to the stride, which is the distance of the robot locomotion in a gait cycle. Two major directions, vertical and horizontal directions are considered here. 

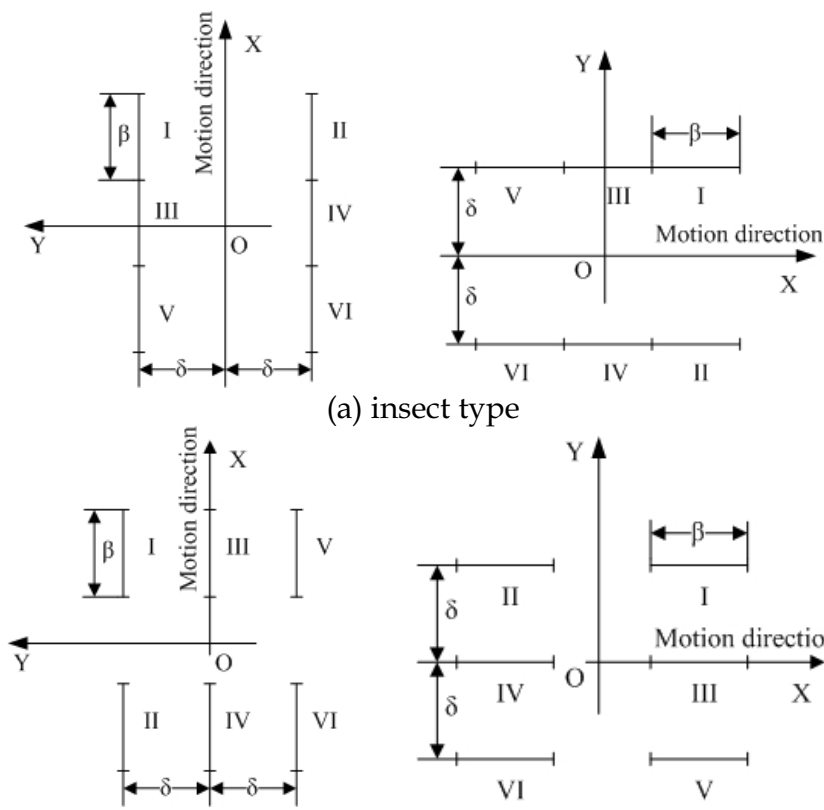

(a) insect type

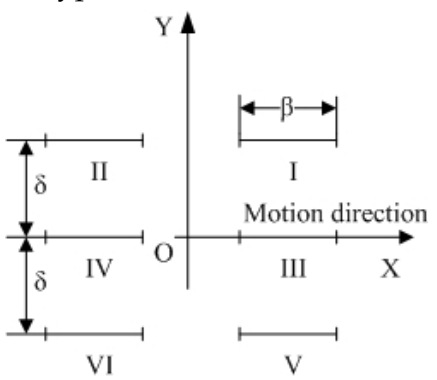

(b) crab type

Figure 14. Two typical geometric models in two climbing directions

As support patterns change in a specific wall gait cycle, the AOD of a gait is defined as the minimum among the various $A O D^{\prime}$ s of the support patterns.

$$
D_{G T}=\min \left(D_{s p i}\right)
$$

When $D_{G T}$ is used as the goal function of optimization approaches, the optimization problem is expressed as

Problem: $\max D_{G T}\left(\phi_{2}, \phi_{3}, \cdots \phi_{6}, \beta\right)$

where $\beta$ is the duty factor of regular periodic gaits, and $\phi_{2}, \phi_{3}, \cdots \phi_{6}$ are relative phases of respective legs.

\section{Results and discussions}

Some results of AOD-optimal gaits are listed in Table2 and Table3. Depicted in Fig. 15 and Fig. 16 are relationships of optimal gaits' $A O D$ varying with duty factor, which clearly show the comparison of different leg stroke layouts, We have discussions as follows:

i) The AOD of optimal gait is proportional to duty factor.

ii) For the insect-type robot climbing in the horizontal direction, or for a crab-type robot climbing in the vertical direction, there is an abrupt increase in AOD of optimal gaits while the duty factor $\beta=2 / 3$.This is because that with this duty factor, it is assured that there are always more than two suction cups in support status along the upper side of the robot during a gait cycle.

iii) When $\beta \leq 2 / 3$, the robot with an insect-type stroke layout is apparently better than the robot with a crab-type stroke layout if the robot is climbing in the horizontal direction. If the robot is climbing in the vertical direction, the situation is visa versa. 


\begin{tabular}{|c|c|c|c|c|c|c|c|}
\hline$\beta$ & $\phi_{2}^{*}$ & $\phi_{3}^{*}$ & $\phi_{4}^{*}$ & $\phi_{5}^{*}$ & $\phi_{6}^{*}$ & $C_{G T}$ & $C_{A V}$ \\
\hline \multirow{5}{*}{$6 / 12$} & 0 & $1 / 2$ & $1 / 4$ & $1 / 2$ & $3 / 4$ & \multirow{5}{*}{1.00} & \multirow{5}{*}{1.50} \\
\hline & $1 / 2$ & $1 / 2$ & 0 & 0 & $1 / 2$ & & \\
\hline & $2 / 3$ & $5 / 6$ & $1 / 6$ & $1 / 3$ & $1 / 2$ & & \\
\hline & $3 / 4$ & 0 & $1 / 4$ & $1 / 2$ & $1 / 2$ & & \\
\hline & $5 / 6$ & $1 / 2$ & $1 / 6$ & $1 / 3$ & $2 / 3$ & & \\
\hline $7 / 12$ & $1 / 2$ & $1 / 2$ & 0 & 0 & $1 / 2$ & 1.17 & 1.43 \\
\hline \multirow{2}{*}{$8 / 12$} & \multirow{2}{*}{0} & \multicolumn{2}{|c|}{$1 / 3$} & \multicolumn{2}{|c|}{$2 / 3$} & \multirow{2}{*}{2.67} & \\
\hline & & \multicolumn{2}{|c|}{$2 / 3$} & \multicolumn{2}{|c|}{$1 / 3$} & & \\
\hline $9 / 12$ & \multirow[b]{3}{*}{0} & \multirow{3}{*}{\multicolumn{2}{|c|}{$\begin{array}{l}1-\beta \leq \phi_{3}^{*} \leq \beta \\
\phi_{4}^{*}=\phi_{3}^{*}\end{array}$}} & \multirow{3}{*}{\multicolumn{2}{|c|}{$\begin{array}{l}\phi_{5}^{*}=\phi_{6}^{*} \\
\text { if } 1-\beta \leq \phi_{3}^{*} \leq 2(1-\beta) \\
1-\beta+\phi_{3}^{*} \leq \phi_{5}^{*} \leq \beta \\
\text { if } 2(1-\beta) \leq \phi_{3}^{*} \leq 2 \beta-1 \\
1-\beta \leq \phi_{5}^{*} \leq \beta-1+\phi_{3}^{*} \\
\text { and } 1-\beta+\phi_{3}^{*} \leq \phi_{5}^{*} \leq \beta \\
\text { if } 2 \beta-1 \leq \phi_{3}^{*} \leq \beta \\
1-\beta \leq \phi_{5}^{*} \leq \beta-1+\phi_{3}^{*}\end{array}$}} & 3.00 & \\
\hline $10 / 12$ & & & & & & 3.33 & \\
\hline $11 / 12$ & & & & & & 3.66 & \\
\hline
\end{tabular}

Table 2. AOD-optimal gaits(robot with insect configuration climbing horizontally) ${ }^{*} D_{A V}$ denotes the average of AOD for successive support patterns

\begin{tabular}{|c|c|c|c|c|c|c|c|}
\hline$\beta$ & $\phi_{2}^{*}$ & $\phi_{3}^{*}$ & $\phi_{4}^{*}$ & $\phi_{5}^{*}$ & $\phi_{6}^{*}$ & $C_{G T}$ & $C_{A V}$ \\
\hline \multirow{2}{*}{$\begin{array}{c}6 / 12 \text { or } \\
7 / 12\end{array}$} & $4 / 3-\beta$ & $1 / 3$ & $2 / 3-\beta$ & $2 / 3$ & $1-\beta$ & 1.17 & 1.17 \\
\hline & $4 / 3-\beta$ & $2 / 3$ & $1-\beta$ & $1 / 3$ & $2 / 3-\beta$ & 1.42 & 1.48 \\
\hline \multirow{2}{*}{$8 / 12$} & 0 & \multicolumn{2}{|c|}{$1 / 3$} & \multicolumn{2}{|c|}{$2 / 3$} & \multirow{2}{*}{2.67} & \multirow{2}{*}{2.67} \\
\hline & 0 & \multicolumn{2}{|c|}{$2 / 3$} & \multicolumn{2}{|c|}{$1 / 3$} & & \\
\hline $9 / 12$ & & \multirow{2}{*}{\multicolumn{2}{|c|}{$\begin{array}{l}1-\beta \leq \phi_{3}^{*} \leq \beta \\
\phi_{4}^{*}=\phi_{3}^{*}\end{array}$}} & \multirow{2}{*}{\multicolumn{2}{|c|}{$\begin{array}{l}\phi_{5}^{*}=\phi_{6}^{*} \\
\text { if } 1-\beta \leq \phi_{3}^{*} \leq 2(1-\beta) \\
1-\beta+\phi_{3}^{*} \leq \phi_{5}^{*} \leq \beta \\
\text { if } 2(1-\beta) \leq \phi_{3}^{*} \leq 2 \beta-1 \\
1-\beta \leq \phi_{5}^{*} \leq \beta-1+\phi_{3}^{*} \\
\text { and } 1-\beta+\phi_{3}^{*} \leq \phi_{5}^{*} \leq \beta \\
\text { if } 2 \beta-1 \leq \phi_{3}^{*} \leq \beta \\
1-\beta \leq \phi_{5}^{*} \leq \beta-1+\phi_{3}^{*}\end{array}$}} & 3.00 & \\
\hline $10 / 12$ & 0 & & & & & 3.33 & 3.89 \\
\hline \multirow{2}{*}{$11 / 12$} & 0 & $5 / 6$ & $1 / 4$ & $1 / 6$ & $1 / 12$ & \multirow{2}{*}{3.89} & \multirow{2}{*}{4.26} \\
\hline & $11 / 12$ & $2 / 3$ & $1 / 12$ & & & & \\
\hline
\end{tabular}

Table 3. AOD-optimal gaits(robot with crab configuration climbing vertically) ${ }^{*} D_{A V}$ denotes the average of AOD for successive support patterns 


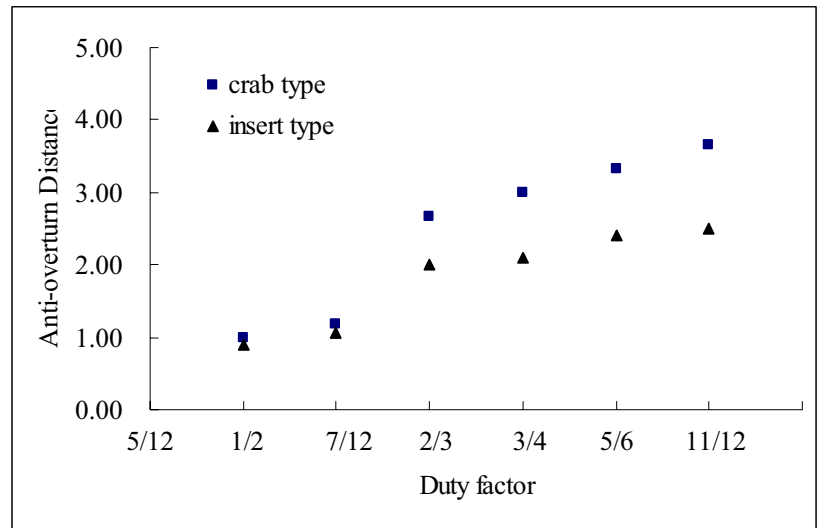

Figure 15. Comparison of AOD with two robot configurations while the wall robot vertically

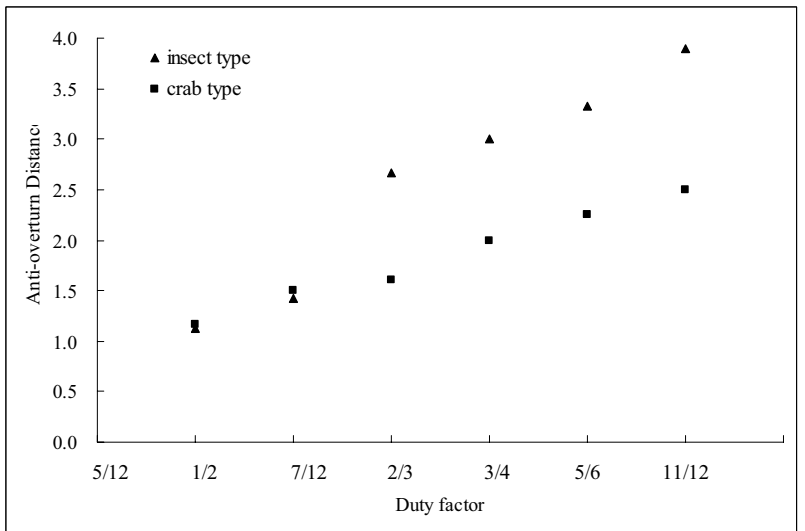

Figure 16. Comparison of AOD with two robot configurations while climbing horizontally

\subsection{Ceiling gait analysis}

While the robot is climbing on an inclined wall surface, the situation is somewhat different from that on the vertical wall surface. We propose another criterion to evaluate the climbing gait.

Anti-overturn Coefficient

Definition 2: Anti-overturn Coefficient (abbreviated as AOC) of a support pattern for a legged robot climbing on an inclined wall surface, $C_{s p}$,is the maximal weight that the robot can carry, $W_{\max }$, divided by suction force of a single sucker, $F_{s}$,

$$
C_{s p}=\frac{W_{\max }}{F_{s}}
$$

Anti-overturn Coefficient of a gait is the minimal of Anti-overturn Coefficient for all support patterns in a gait cycle.

$$
C_{G T}=\left(C_{s p i}\right)_{\min }
$$


For a legged robot climbing on the ceiling as is depicted in Fig.17,AOC is calculated by the following theorem.

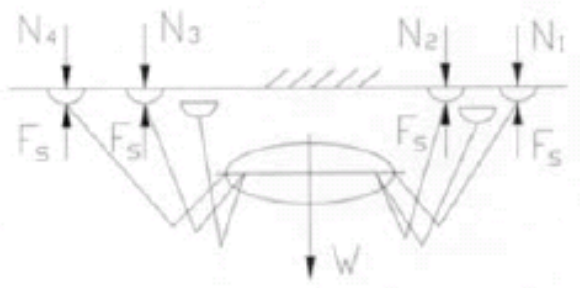

Fig.17. Legged robot climbing on ceilings

Theorem 2: For a legged robot climbing on a horizontal Ceiling, Anti- overturn Coefficient of a specific support pattern,is calculated as

$$
C_{s p}=-1 / \min _{i=1 \sim n}\left(r_{0 i}^{\prime}\right)
$$

where $n$ is the number of suckers in support status, $r_{0 i}$ is the $i$ th element of vector $\mathbf{R}_{0}^{\prime}$;

$$
\mathbf{R}_{0}^{\prime}=\mathbf{A}^{-1} \mathbf{Q}_{0}^{\prime}
$$

where $\mathbf{A}$ is the same matrix as Eq.(4), while $\mathbf{Q}_{\mathbf{0}}^{\prime}$ is the unit load vector normalized to $W$,

$$
\mathbf{Q}_{0}^{\prime}=\left\{\begin{array}{c}
-1 \\
0 \\
0 \\
\vdots \\
0
\end{array}\right\}_{n \times 1}
$$

\section{Proof:}

i) We begin with the general scenario of the robot adhering on inclined wall surface with angle $\theta$,the following matrix equation still stands,

$$
\mathbf{A}(\mathbf{N}-\mathbf{F})=\mathbf{Q}^{\prime}
$$

Where $\mathbf{A}$ is the same matrix as Eq.(4),while $\mathbf{Q}^{\prime}$ is as follows,

$$
\mathbf{Q}^{\prime}=\left\{\begin{array}{c}
-W \cos \theta \\
-W \sin \alpha \cdot \sin \theta \cdot H \\
-W \cos \alpha \cdot \sin \theta \cdot H \\
\vdots \\
0
\end{array}\right\}_{n \times 1}
$$

This time we denote

$$
\mathbf{N}=W \mathbf{N}_{0}^{\prime}, \mathbf{Q}^{\prime}=W \mathbf{Q}_{0}^{\prime}
$$


and substitute them into $\mathrm{Eq}(25)$

$$
\mathbf{A}\left(W \mathbf{N}_{0}^{\prime}-F_{s} \mathbf{I}\right)=W \mathbf{Q}_{0}^{\prime}
$$

We can get

$$
\mathbf{N}_{0}^{\prime}=\frac{F_{s}}{W} \mathbf{I}+\mathbf{A}^{-1} \mathbf{Q}_{0}^{\prime}
$$

Denote

$$
\mathbf{R}_{0}^{\prime}=\mathbf{A}^{-1} \mathbf{Q}_{0}^{\prime}
$$

Substitute it into $\mathrm{Eq}(28)$

$$
\mathbf{N}_{\mathbf{0}}^{\prime}=\frac{F_{s}}{W} \mathbf{I}+\mathbf{R}_{\mathbf{0}}^{\prime}
$$

When $W$ increase and approaches $W_{\max }$, the minimum of the normal reaction forces acting on suckers becomes zero,

$$
\min _{i=1 \sim n}\left(N_{0 i}^{\prime}\right)=0
$$

We can extract an algebra equation from $\mathrm{Eq}(30)$

and

$$
0=\frac{F_{s}}{W_{\max }}+\min _{i=1 \sim n}\left(r_{0 i}^{\prime}\right)
$$

$$
C_{s p}=\frac{W_{\max }}{F_{s}}=-\frac{1}{\min _{i=1 \sim n}\left(r^{\prime}{ }_{0 i}\right)}
$$

ii) For the situation of standard ceiling when $\theta=0$, Eq.(26) becomes

And

$$
\mathbf{Q}^{\prime}=\left\{\begin{array}{c}
-W \\
0 \\
0 \\
\vdots \\
0
\end{array}\right\}_{n \times 1}
$$

$$
\mathbf{Q}_{\mathbf{0}}^{\prime}=\frac{\mathbf{Q}^{\prime}}{W}=\left\{\begin{array}{c}
-1 \\
0 \\
0 \\
\vdots \\
0
\end{array}\right\}_{n \times 1}
$$

\section{End of Proof}

It is cleat that Anti-overturn Coefficient is a measurement of overturn-resistance ability of a robot in a definite effective body height $\mathrm{H}$. And it has relation with AOD,

$$
D_{s p}=H \cdot C_{s p}
$$




\section{Optimization of ceiling gait}

Employing the gait $\mathrm{AOC}$ as an optimization goal function the optimal relative phases for regular periodic ceiling are computed, for different duty factor. The result are listed in Table 4 and Table 5. It is noted that there is no significant difference for the insect-type and the crab-type of leg stroke layouts.

\begin{tabular}{|c|c|c|c|c|c|c|c|}
\hline$\beta$ & $\phi_{2}^{*}$ & $\phi_{3}^{*}$ & $\phi_{4}^{*}$ & $\phi_{5}^{*}$ & $\phi_{6}^{*}$ & $C_{G T}$ & $C_{A V}$ \\
\hline \multirow{2}{*}{$6 / 12$} & $1 / 2$ & $1 / 6$ & $5 / 6$ & $2 / 3$ & $1 / 3$ & \multirow{2}{*}{1.71} & \multirow{2}{*}{1.91} \\
\hline & $2 / 3$ & $1 / 2$ & $1 / 6$ & $1 / 3$ & $5 / 6$ & & \\
\hline \multirow{2}{*}{$7 / 12$} & $1 / 2$ & $2 / 3$ & $11 / 12$ & $1 / 2$ & $1 / 4$ & \multirow{2}{*}{2.00} & \multirow{2}{*}{2.74} \\
\hline & $3 / 4$ & $1 / 6$ & $5 / 12$ & $1 / 2$ & 0 & & \\
\hline \multirow{2}{*}{$8 / 12$} & $1 / 3$ & $1 / 3$ & $2 / 3$ & $1 / 3$ & 0 & \multirow{2}{*}{2.97} & \multirow{2}{*}{3.03} \\
\hline & $2 / 3$ & 0 & $1 / 3$ & $2 / 3$ & 0 & & \\
\hline \multirow{2}{*}{$9 / 12$} & $5 / 12$ & $2 / 3$ & $2 / 3$ & $1 / 4$ & 0 & \multirow{2}{*}{3.24} & \multirow{2}{*}{3.76} \\
\hline & $3 / 4$ & $5 / 12$ & $5 / 12$ & $3 / 4$ & $1 / 6$ & & \\
\hline \multirow{4}{*}{$10 / 12$} & $5 / 12$ & $2 / 3$ & $3 / 4$ & $5 / 12$ & $1 / 12$ & \multirow{4}{*}{3.54} & \multirow{4}{*}{4.31} \\
\hline & $1 / 2$ & $3 / 4$ & $5 / 6$ & $1 / 2$ & $1 / 4$ & & \\
\hline & $2 / 3$ & $1 / 4$ & $1 / 3$ & $7 / 12$ & 0 & & \\
\hline & $3 / 4$ & $1 / 4$ & $1 / 3$ & $1 / 2$ & 0 & & \\
\hline \multirow{2}{*}{$11 / 12$} & $1 / 3$ & $5 / 12$ & $3 / 4$ & $1 / 3$ & 0 & \multirow{2}{*}{3.92} & \multirow{2}{*}{4.65} \\
\hline & $2 / 3$ & $5 / 12$ & $5 / 12$ & $2 / 3$ & 0 & & \\
\hline
\end{tabular}

Table 4. Optimal ceiling gaits for insect-type robot

${ }^{*} C_{A V}$ denotes the average of AOD for successive support patterns

\begin{tabular}{|c|c|c|c|c|c|c|c|}
\hline$\beta$ & $\phi_{2}^{*}$ & $\phi_{3}^{*}$ & $\phi_{4}^{*}$ & $\phi_{5}^{*}$ & $\phi_{6}^{*}$ & $C_{G T}$ & $C_{A V}$ \\
\hline \multirow{7}{*}{$6 / 12$} & $1 / 4$ & $3 / 4$ & $1 / 2$ & $1 / 2$ & 0 & \multirow{7}{*}{2.00} & \multirow{7}{*}{2.00} \\
\hline & $1 / 3$ & $5 / 6$ & $1 / 2$ & $1 / 2$ & 0 & & \\
\hline & $5 / 12$ & $11 / 12$ & $1 / 2$ & $1 / 2$ & 0 & & \\
\hline & $1 / 2$ & 0 & $1 / 2$ & $1 / 2$ & 0 & & \\
\hline & $7 / 12$ & $1 / 12$ & $1 / 2$ & $7 / 12$ & $1 / 12$ & & \\
\hline & $2 / 3$ & $1 / 6$ & $1 / 2$ & $2 / 3$ & $1 / 6$ & & \\
\hline & $3 / 4$ & $1 / 4$ & $1 / 2$ & $3 / 4$ & $1 / 4$ & & \\
\hline $7 / 12$ & $1 / 2$ & $1 / 2$ & 0 & 0 & $1 / 2$ & 2.00 & 3.70 \\
\hline $8 / 12$ & $1 / 2$ & $2 / 3$ & $1 / 6$ & $1 / 3$ & $5 / 6$ & 2.67 & 3.17 \\
\hline $9 / 12$ & $1 / 2$ & $1 / 2$ & 0 & $3 / 4$ & $1 / 4$ & 3.24 & 2.39 \\
\hline \multirow{2}{*}{$10 / 12$} & $1 / 3$ & $1 / 3$ & $1 / 6$ & $5 / 6$ & 0 & \multirow{2}{*}{3.66} & \multirow{2}{*}{3.92} \\
\hline & $2 / 3$ & $5 / 6$ & 0 & $2 / 3$ & $1 / 2$ & & \\
\hline \multirow{2}{*}{$11 / 12$} & $1 / 3$ & $1 / 3$ & $1 / 4$ & $11 / 12$ & 0 & \multirow{2}{*}{3.88} & \multirow{2}{*}{4.21} \\
\hline & $2 / 3$ & $11 / 12$ & 0 & $2 / 3$ & $7 / 12$ & & \\
\hline
\end{tabular}

Table 5 Optimal ceiling gaits for insect-type robot

*There are altogether 33 optimal gaits for $\beta=6 / 12$, only some are listed in this table 


\section{Conclusion}

The research on transition gait programming makes it possible for the robot to act both as a ground-walking machine and as a wall-climbing robot as well. It forms the basis of gait kinematics in developing a six-legged wall-and-ground-walking robot.

It is a new attempt to investigate ground-to-wall transit gait programming of six-legged robot in the research field of walking machines. Employing kinematics geometry, the authors decompose the relatively complicated transit walking into combination of two basic motions. The method proposed is verified by kinematics simulation as correct and effective. The feasibility of the ground-wall transitional motion of the legged robot is mainly determined by the distance $\mathrm{H}$ and $\mathrm{D}$. Too large or small values of them always lead to the failure of the motion. And the length $\mathrm{L}$ of the robot body is the key of the rapidity of the ground-wall transition motion, it should be assigned relative small value in the permissible limitation of the structure design.

Based on the reasonable selection for various parameters, the times of adjusting the robot body can be decrease to four, which is very effective.

Anti-overturn Distance (AOD) is a geometric measurement proposed to evaluate overturnresistance capability, which is solely dependent on the support pattern of wall gaits. AOD is as important to wall gait programming as the stability margin is to ground gaits. While the robot is climbing on an inclined wall surface or ceilings, a related measurement, Antioverturn Coefficient, is used to evaluate ceiling gaits.

Optimal regular periodic wall gaits and ceiling gaits are obtained employing both Antioverturn Distance and Anti-overturn Coefficient. In most cases, several optimal gaits exist for each given duty factor.

For a six-legged robot climbing on a vertical wall, crab-type leg-stroke layout is preferred for climbing vertically; insect-type leg-stroke layout is preferred far climbing horizontally. If one robot is going to walk in bath directions, it is required that the robot can change its legstroke layout, which is possible if the robot is designed to have a 3-D leg configuration. While for a six-legged robot climbing on ceilings, there is no significant difference between the two leg-stroke types.

Considering both the climbing safety and the climbing speed, the duty factor of wall gaits recommended is $2 / 3$.

\section{Acknowledgments}

This research is partly sponsored by the Robotic Mechanism Laboratory under the National High-tech R\&D Program of China.

\section{References}

Bessonov, A., and Umnov, N.,1973, The Analysis of Gaits in Six-legged Vehicle According Their Static Stability, Proceedings, 1st Symposium on Theory and Practice of Robots and Manipulators, pp.1-10.

Chen, Z., Gong., Z, Qian, J., et al, 1997, Research on robotic Wall-climbing Mechanism, Journal of Shanghai University, Vol. 3 No.6, pp.663-669. (in Chinese)

Hirose, S., et al., 1992, Legged Wall-climbing Robot, Journal of Japanese Robot Association, Vol. 10, No. 5, pp.575-580 (in Japanese) 
Hirose. S., 1984, A Study of Design and Control of a Quadruped Walking Vehicle, International Journal of Robotics Research, Vol. 3 No. 2, pp.113-133

Ikeda, K., et al., 1989, Basic Study on a Wall Climbing Robot(II), Journal of Mechanical Engineering Laboratory, Vol. 43. No. 4, pp.25-33 (in Japanese)

Kroczynski P., et al., 1987, The Skywasher: a Building Washing Robot, Proceedings, Proceedings, 17th International Symposium of Industrial Robots, pp.1.11-1.19

Luk, B. L., et al., 1991, ROBUG II: an Intelligent Wall Climbing Robot, Proceedings, Proceedings, IEEE International Symposium on Robotics and Automation, pp.2342-2347

McGhee, R. B., Frank A. A., 1968, On the Stability Properties of Quadruped Creeping Gaits, Mathematical Bioscience, Vol. 3, No. 3, pp.331-351

Men, G., Zhao, and Y., Wang Y, 1994, The Control System of Wall Climbing Robot, Proceedings, 2nd Asian Conference on Robotics and Its Applications, Beijing, pp.375377

Nagastuka, K., 1986, Vacuum Adhering Crawler System 'VACS', Robot, No. 53, pp.127135 (in Japanese)

Nishi, A., et al., 1992, Development of a Wall-climbing Robot, Robot, No. 84, pp.111-116. (in Japanese)

Qian, J., Gan, D., 1988, Stability Study of Six Legged Laterally Walking Gaits, Proceedings, ASME Design Engineering Conference, Vol.15-3 PT3, pp.171-176

Ikeda, K., Nozaki, T., 1991, Development of a Self-contained Wall-climbing Robot, Proceedings, International Symposium on Advanced Robot Technology, pp.356-372

Sato, K. Morita, H., 1992, On-Wall Locomotion Technology for Advanced Robot Technology Research, Japan Robot Society Journal, Vol. 10, No.5, pp.581-589 (in Japanese)

Hirose, S., Nagalobo, A., 1992, Legged Wall-climbing Robot, Japan Robot Society Journal, Vol. 10, No. 5, pp.19-24 (in Japanese)

Qian, J., Gan D., 1992, Stability-optimized Gait Series for Omni-directional Hexapods While Walking Laterally, Journal of Shanghai University of Sciences and Technology, Vol. 15, No. 3, pp.60-67 (in Chinese)

Qian, J., Gong Z., et al., 1994, Anti-Overturn Distance and Its Applications in Multi-legged Wall-climbing Robots, Proceedings, 2nd.Asian Conference on Robotics and Its Application, pp.318-322

Gu, J., Qian, J., et al., 1997, Study on Feasibility and Rapidty of the Ground-wall Transitional of Legged Robot, Proceedings, 2nd China-Japan Mechatronics Symposium, Chengdu, China

Qian, J., 1993, On the Gait Kinematics of Six-legged Robot Capable of Ground and Wall Walking, Ph. D Dissertation of Beijing University of Aeronautics and Astronautics (in Chinese) 


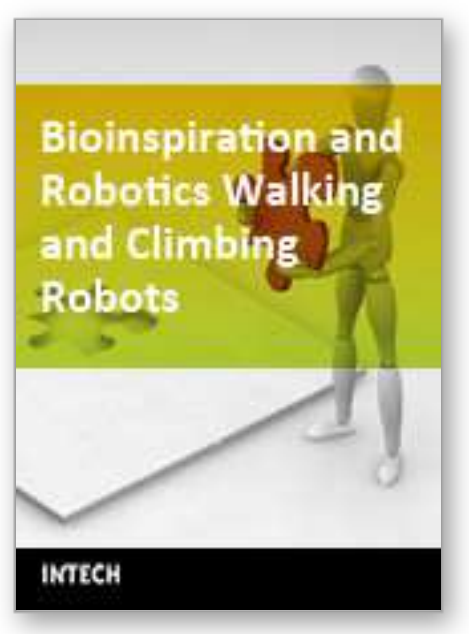

\section{Bioinspiration and Robotics Walking and Climbing Robots \\ Edited by Maki K. Habib}

ISBN 978-3-902613-15-8

Hard cover, 544 pages

Publisher I-Tech Education and Publishing

Published online 01, September, 2007

Published in print edition September, 2007

Nature has always been a source of inspiration and ideas for the robotics community. New solutions and technologies are required and hence this book is coming out to address and deal with the main challenges facing walking and climbing robots, and contributes with innovative solutions, designs, technologies and techniques. This book reports on the state of the art research and development findings and results. The content of the book has been structured into 5 technical research sections with total of 30 chapters written by well recognized researchers worldwide.

\section{How to reference}

In order to correctly reference this scholarly work, feel free to copy and paste the following:

Jinwu Qian, Zhen Zhang and Li Ma (2007). Gait Programming for Multi-Legged Robot Climbing on Walls and Ceilings, Bioinspiration and Robotics Walking and Climbing Robots, Maki K. Habib (Ed.), ISBN: 978-3-90261315-8, InTech, Available from:

http://www.intechopen.com/books/bioinspiration_and_robotics_walking_and_climbing_robots/gait_programmin g_for_multi-legged_robot_climbing_on_walls_and_ceilings

\section{INTECH}

open science | open minds

\author{
InTech Europe \\ University Campus STeP Ri \\ Slavka Krautzeka 83/A \\ 51000 Rijeka, Croatia \\ Phone: +385 (51) 770447 \\ Fax: +385 (51) 686166 \\ www.intechopen.com
}

\author{
InTech China \\ Unit 405, Office Block, Hotel Equatorial Shanghai \\ No.65, Yan An Road (West), Shanghai, 200040, China \\ 中国上海市延安西路65号上海国际贵都大饭店办公楼 405 单元 \\ Phone: +86-21-62489820 \\ Fax: $+86-21-62489821$
}


(C) 2007 The Author(s). Licensee IntechOpen. This chapter is distributed under the terms of the Creative Commons Attribution-NonCommercial-ShareAlike-3.0 License, which permits use, distribution and reproduction for non-commercial purposes, provided the original is properly cited and derivative works building on this content are distributed under the same license. 